$\xi_{p-1}$

\title{
Dielectric Studies of PEO-based Polymer Electrolytes Doped with LiTFSI Salt and EMIMTFSI Ionic Liquid
}

\author{
Nor Hayati Al-Madihah Hashim ${ }^{1 *}$, Ri Hanum Yahaya Subban ${ }^{1,2}$ \\ ${ }^{1}$ Faculty of Applied Sciences, Universiti Teknologi MARA, 40450 Shah Alam, Selangor, Malaysia \\ ${ }^{2}$ Institute of Science, Universiti Teknologi MARA, 40450 Shah Alam, Selangor, Malaysia \\ *Corresponding author E-mail: Hayati.almadihah@gmail.com
}

\begin{abstract}
In this work, poly (ethylene oxide) (PEO) is doped with Lithium bis (trifluoromethanesulfonyl) imide (LiTFSI) salt and 1-Ethyl-3methylimidazolium bis (trifluromethanesulfonyl) imide (EMIm-TFSI) ionic liquid, a plasticiser, for preparation of PEO-LiTFSIEMImTFSI plasticised polymer electrolyte (PPE) system. Impedance spectroscopy is used to analyse the dependence of dielectric behaviour of the films on frequency and temperature. It is performed in the temperature range between $303 \mathrm{~K}$ to $343 \mathrm{~K}$ for frequencies between $50 \mathrm{~Hz}$ to $1 \mathrm{MHz}$. The cationic transport in the films may be explained from the dielectric constant, $\varepsilon_{\mathrm{r}}$ and dielectric loss, $\varepsilon_{\mathrm{i}}$ where they are found to increase with temperature at lower frequency and decrease at higher frequency, showing that the electrodes were being polarized. Both real parts, $\mathrm{M}_{\mathrm{r}}$ and imaginary part, $\mathrm{M}_{\mathrm{i}}$ of the modulus showed an increase at high frequency that indicates the spread of conductivity relaxation. The highest temperature $(343 \mathrm{~K})$ achieved the lowest relaxation time, $\tau$ due to increase in number of ions and increase in mobility of ions in the polymer system indicating that conductivity in this system is thermally activated.
\end{abstract}

Keywords: Polymer electrolyte, Impedance spectroscopy, Dielectric properties, Electric modulus, Relaxation time.

\section{Introduction}

The demand for lithium ion batteries has been growing tandem globally. However, the conventional application for lithium ion batteries are limited due to the use of liquid organic electrolyte [1]. They suffer from safety problems because of their flammable nature. The conventional electrolyte can easily catch fire and even be explosive [1] if there are any short circuits, local overheating or misuse. Hence, polymer electrolyte, which is solid in nature, has been introduced as an alternative to replace the conventional liquid electrolyte. It has been widely studied and has been shown to be safe for use due to no leakage and overheating.

There are three different types of polymer electrolyte. Dry polymer electrolyte, plasticized/ gel polymer electrolyte (PPE) and composite polymer electrolyte [2]. PPE have received a lot of attention in recent years, as it possesses both cohesive property of solids and diffusive property of liquids. This hybrid system is formed by trapping a large amount of liquid electrolyte as lithium ion conducting organic solution in a polymer matrix [1]. This unique characteristic makes PPE garner various important applications [3] including as electrolyte in lithium batteries.

The main important component of a polymer electrolyte is the choice of polymer host. PEO has been studied widely as a polymer host due to its ability to coordinate ionic species and its local flexibility that allows for ionic mobility [4] and hence has been chosen in this work. Other important properties are its high melting point, good structure integrity and low toxicity [5].

Unfortunately, despite the advantages, some drawbacks are present which includes higher crystallinity at lower temperature resulting in lower room temperature ionic conductivity of the electrolytes (typically $\leq 10^{-5} \mathrm{~cm}^{-1}$ ). Hence, in this work, LiTFSI salt is doped into PEO since it has a bulky anion, which suppresses crys- tallization of the polymer [6] and it has low dissociation energy so that it can easily be dissociated into ions, which in turn contribute to ionic conductivity enhancement.

The polymer electrolyte PEO-LiTFSI is also incorporated with ionic liquid as plasticizer, which is thought to enhance both ionic conductivity and safety of the polymer electrolyte at the same time [7]. The ionic liquid helps to dissociate more salt leading to higher ionic conductivity since it increases the amorphous region, dissociate ion aggregates and lowers the glass transition tempearature [8]. Also since the anion of the ionic liquid chosen is the same as the anion of the doping salt, cross contact ion pairs may be prevented.

The aim of this paper is to study the dependence of dielectric properties of PEO-LiTFSI-EMImTFSI polymer electrolyte system on frequency and temperature. This dependence is analysed using Impedance Spectroscopy for frequencies in the range of $50 \mathrm{~Hz}-1$ $\mathrm{MHz}$ and for temperatures between $303 \mathrm{~K}$ to $343 \mathrm{~K}$ to understand the effects of temperature towards the conductivity of the PPE system studied in this work.

Impedance spectroscopy is a powerful tool usually employed to obtain the conductivity value of the polymer electrolyte. It is a useful technique for studying various aspects of solid ionics. Besides its versatility and simplicity, impedance spectroscopy also has its limitations. Relatively expensive equipment is required. Interpretation of data requires a good understanding of A.C. impedance theory. Care must also be taken in analyzing the impedance data to avoid the possibility of false interpretation of the data. 


\section{Experimental}

\subsection{Materials}

PEO (Sigma-Aldrich, average molecular weight ca. $8000000 \mathrm{~g}$ mol $\left.^{-1}\right)$, EMIm-TFSI $\left(\mathrm{C}_{8} \mathrm{H}_{11} \mathrm{~F}_{6} \mathrm{~N}_{3} \mathrm{O}_{4} \mathrm{~S}_{2}\right)$ (Sigma-Aldrich and Methanol solution were purchased from Labchem Sdn. Bhd. Lithium salt, LiTFSI $\left(\mathrm{LiCF}_{3} \mathrm{SO}_{3}\right.$ ) (Sigma-Aldrich) was received from ICC Lab, Faculty of Applied Sciences, Universiti Teknologi MARA.

\subsection{Preparation of Plasticised Polymer Electrolyte Film}

$1 \mathrm{~g}$ of PEO was dissolved in $50 \mathrm{ml}$ methanol solution at $50^{\circ} \mathrm{C}$ Once it is fully dissolved, $30 \mathrm{wt}$. \% of LiTFSI (following the equation xPEO - [1-x] LiTFSI, where $\mathrm{x}$ is wt. \% of PEO) is added into the PEO solution and stirred for 24 hours at $50^{\circ} \mathrm{C}$ until a homogenous solution is obtained. Then, $8 \mathrm{wt}$. \% of EMImTFSI (following the equation 1) is added into the PEO-LiTFSI system and stirred for further 24 hours at room temperature [9]. The solution is casted into Teflon petri dishes and allowed to evaporate slowly at room temperature [5]. Flexible and free standing films of thickness between $145-150 \mu \mathrm{m}$ is obtained and is stored in a desiccator for continuous drying.

$$
\text { wt. } \% \text { of } I L=\frac{\text { wt. of ionic liquid }}{w t . \text { of PBO+wt. of lithium salt }+w t \text {. of ionic liquid }} \times 100 \%
$$

\subsection{Characterisation of Polymer Electrolyte Film}

The study on dielectric properties is carried out for the PPE with 8 wt. \% of EMImTFSI (PEIL08) using Impedance Spectroscopy in the frequency range of $50 \mathrm{~Hz}$ to $1 \mathrm{MHz}$ for temperatures between $303 \mathrm{~K}$ to $343 \mathrm{~K}$. The analysis is carried out by sandwiching the PPE between two stainless steel (SS) blocking electrodes with diameter of $1 \mathrm{~cm}$ with configuration SS/PPE/SS. Impedances of the films were measured using the HIOKI $3531 \mathrm{Z} \mathrm{LCR} \mathrm{Hi-tester} \mathrm{impedance}$ meter interfaced to a computer. The impedance data obtained is converted into both dielectric $(\varepsilon)$ and electric modulus (M) formalisms using the following equations:

$\varepsilon_{\mathrm{r}}(\omega)=\mathrm{Z}_{\mathrm{i}} / \omega \mathrm{C}_{\mathrm{o}}\left(\mathrm{Z}_{\mathrm{r}}^{2}+\mathrm{Z}_{\mathrm{i}}^{2}\right)$

$\varepsilon_{\mathrm{i}}(\omega)=\mathrm{Z}_{\mathrm{r}} / \omega \mathrm{C}_{\mathrm{o}}\left(\mathrm{Z}_{\mathrm{r}}^{2}+\mathrm{Z}_{\mathrm{i}}^{2}\right)$

$\mathrm{M}_{\mathrm{r}}(\omega)=\varepsilon_{\mathrm{r}} /\left(\varepsilon_{\mathrm{r}}^{2}+\varepsilon_{\mathrm{i}}^{2}\right)$

$\mathrm{M}_{\mathrm{i}}(\omega)=\varepsilon_{\mathrm{i}} /\left(\varepsilon_{\mathrm{r}}^{2}+\varepsilon_{\mathrm{i}}^{2}\right)$

where $Z_{i}$ and $Z_{r}$ are imaginary and real impedance respectively, $C_{o}$ is vacuum capacitance $\left(C_{0}=\varepsilon_{0} A / t, t\right.$ is thickness of the electrolyte, $\mathrm{A}$ is the surface area of contact, $\varepsilon_{\mathrm{o}}$ is the permittivity of free space) and $\omega$ is the angular frequency, being $2 \pi f$. These formalisms are chosen since they display different aspects of the dielectric behavior; for example, the dielectric formalism (ع) highlights the effects of polarization whereas the electric moduli formalism (M) suppresses the effects of polarization but instead highlights the bulk dielectric behavior.

\section{Results and Discussion}

Dielectric constant, $\varepsilon_{\mathrm{r}}$ also known as the real part of dielectric constant is associated with a material's capacity to store charge, represents the amount of dipole alignment in a given volume and hence is a measure of the material's polarization. Since the number of ions present affects the amount of charge stored, then, if the number of ions increase, the number of charge stored increases. While for imaginary dielectric constant, also known as dielectric loss, $\varepsilon_{\mathrm{i}}$. it is used to measure the dissipation of energy generated from transportation of charge carriers and alignment of dipoles when the polarity of electric field reverses and hence is a measure of polarization effects of charge carriers [10] where it is related to the electrical conductivity of the material.

Fig. 1(a) and (b) shows the dependence of dielectric constant and dielectric loss as a function of frequency at various temperatures for PEIL08. The nature of dielectric permittivity for free dipoles oscillating in an alternating field may be described in the following manner. At very low frequencies $(\omega<1 / \tau, \tau$ is the relaxation time),dipoles follow the field and the value of dielectric constant $\varepsilon_{\mathrm{r}}$ $=\varepsilon_{\mathrm{s}}$ (value of dielectric constant at quasistatic fields) $[4,11]$. As the frequency increases (with $\omega<1 / \tau$ ), the dielectric constant drops (relaxation process). At very high frequencies $(\omega>>1 / \tau)$, dipoles can no longer follow the field and $\varepsilon_{\mathrm{r}}=\varepsilon_{\infty}$ (high frequency value of $\varepsilon_{\mathrm{r}}$ ).

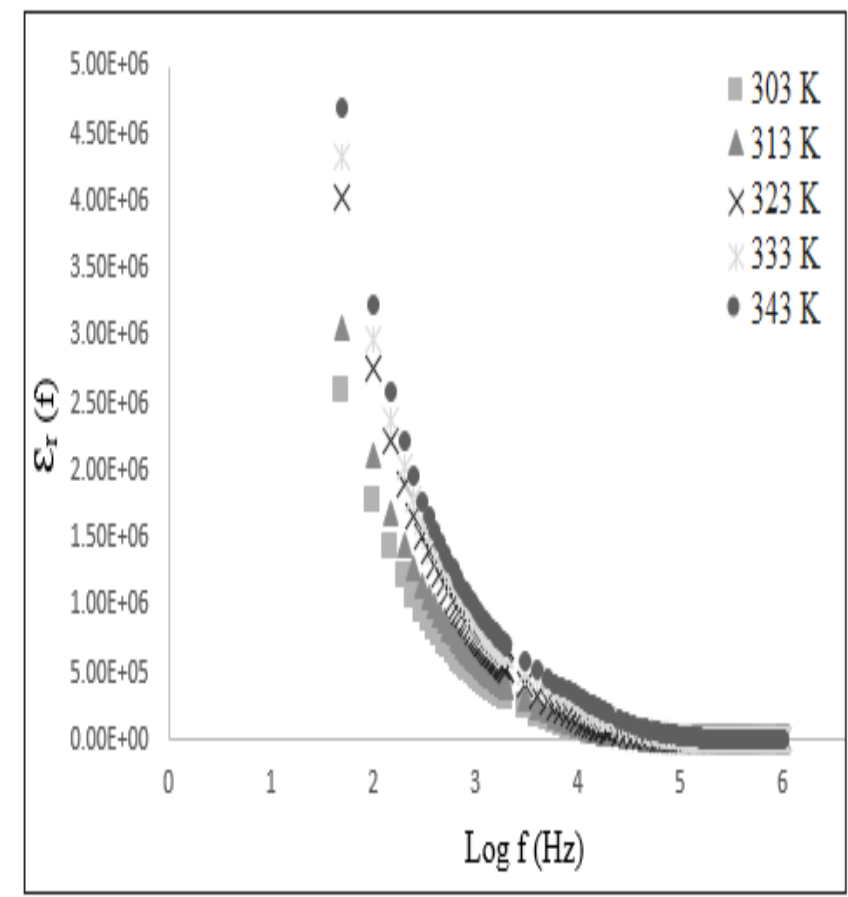

Fig. 1(a): Variation of dielectric constant with $\log$ frequency at various temperatures.

Qualitatively the above described behavior is observed in Fig. 1 (a) and (b). The dielectric constant at low frequency is observed to be rather high, is found to decrease with frequency at first, and then becomes more or less stabilized. The high value of $\varepsilon_{\mathrm{r}}$ at frequencies lower than $100 \mathrm{~Hz}$, which increases with decreasing frequency and increasing temperature, corresponds to bulk effect of the system. Higher value of dielectric constant observed at high temperature and very low frequencies may be attributed to free charge buildup at the interfaces within the bulk of the sample (interfacial polarization) and at the interface between the sample and the electrodes (space-charge polarization) indicating that the sample at $343 \mathrm{~K}$ has the highest amount of charge stored and hence the highest conductivity.

The dielectric loss is also rather high at low frequency but falls quickly with rising frequency. Similar to the dependence of dielectric constant on temperature, the dielectric loss increases with increasing temperature. This indicates the thermally activated nature of dielectric relaxation of the polymer electrolyte system and that heat or temperature increases the degree of salt dissociation and redissociation of ion aggregates and hence increases conductivity due to higher ion content. The thermally activated behaviour is also apparent from the displacement of the rise in both dielectric constant and dielectric loss to higher frequencies at higher temperatures indicating that the number of charges and hence ion present increases with temperature. 


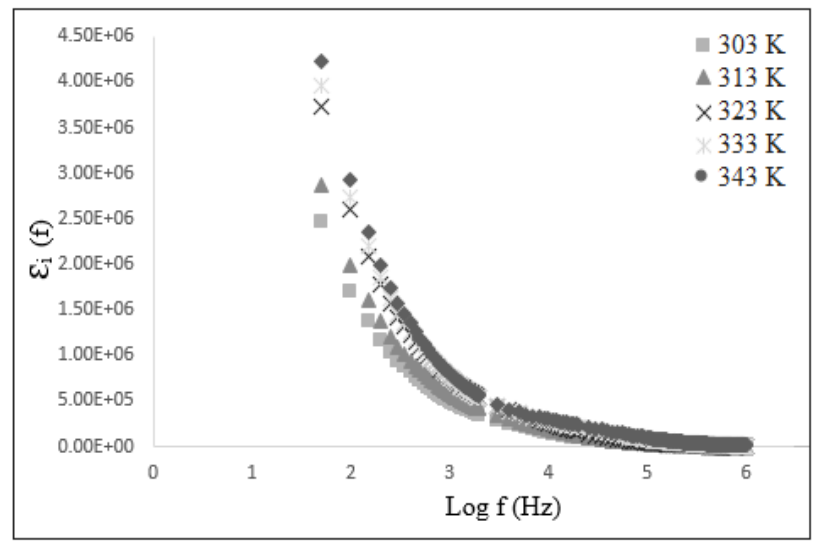

Fig. 1(b): Variation of dielectric loss with $\log$ frequency at various temperatures.

In order to analyse the relaxation processes, dielectric moduli analysis is presented to highlight the bulk dielectric behavior and suppress the effects of electrode polarization [12]. Fig. 2 (a) and (b) depicts both real and imaginary part of the electric moduli at temperatures between $303 \mathrm{~K}$ to $343 \mathrm{~K}$ for PEIL08.

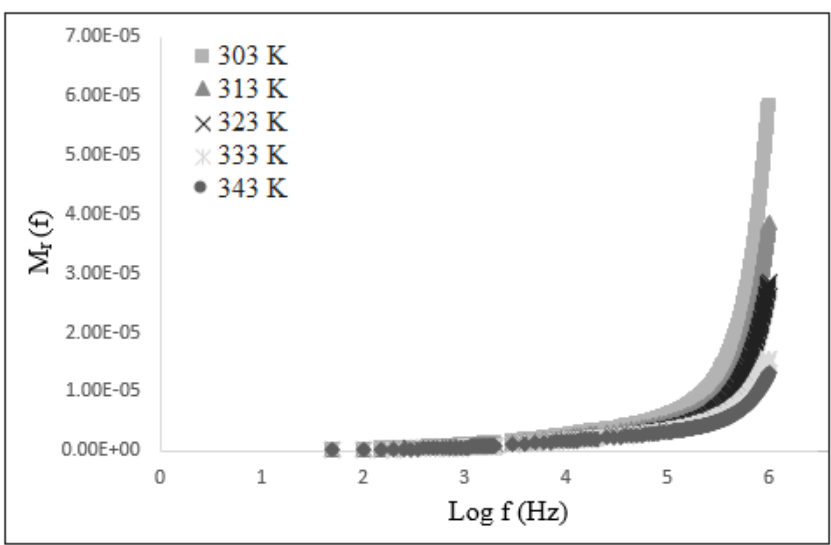

Fig. 2(a): Variation of real part of electric modulus with $\log$ frequency at various temperatures.

At low frequencies, $M_{r}$ and $M_{i}$ approaches zero for all temperatures indicating that electrode polarization phenomenon makes negligible contribution [13]. The presence of the long tail at the low frequency end is attributed to the large capacitance associated with the electrodes [14], which gives added evidence of the suppression of electrode polarization. It can be observed that both $\mathrm{M}_{\mathrm{r}}$ and $\mathrm{M}_{\mathrm{i}}$ have low values at lower frequencies and slowly increase as the frequencies increased. The rise in both $\mathrm{M}_{\mathrm{r}}$ and $\mathrm{M}_{\mathrm{i}}$ is displaced towards higher frequencies at higher temperatures giving indication of a thermally activated behavior implying higher conductivity at higher temperatures. The high value at higher frequencies is due to conductivity relaxation spreading over the range of frequencies studied [15], i.e. the existence of a temperaturedependent electrical relaxation phenomenon in the system. It also indicates that ionic conduction motion and polymer segmental motion are strongly coupled in the system. This implies that as temperature increased, the polymer segmental motion increases giving it more flexibility which in turn aids in the transport of ions. In other words, as the polymer segments become more flexible with temperature the mobility of ion increases and so does the conductivity. This is in line with the very fundamental concept of conductivity described by the equation:

$$
\sigma=n q \mu
$$

Where $\sigma$ is conductivity, $\mathrm{n}$ is number of charge carriers/ions and $\mu$ is the mobility. Also it is to be noted that at higher temperatures $\mathrm{n}$, the number of ions increases as evidenced by the graph in Fig. 1 (a), where the value of $\varepsilon_{\mathrm{r}}$ is the highest at the highest temperature.

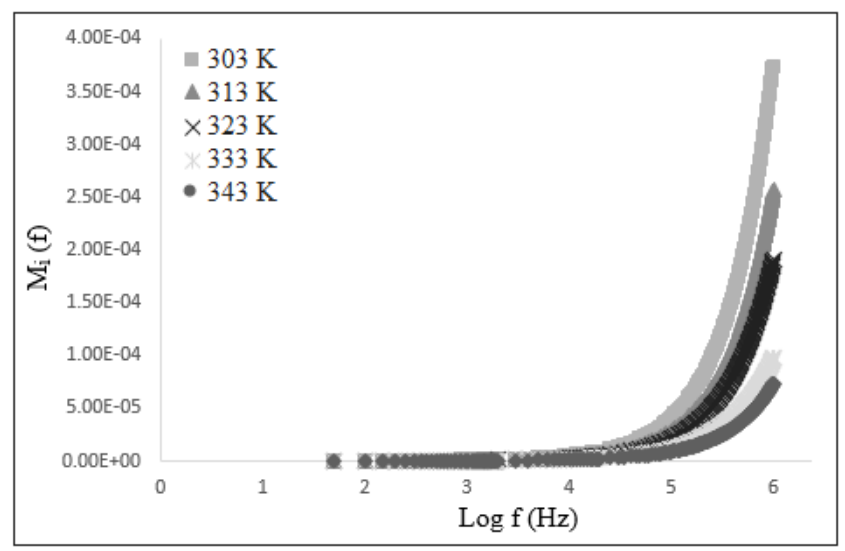

Fig. 2(b): Variation of imaginary part of electric modulus with log frequency at various temperatures.

The relaxation phenomenon may be interpreted from a graph of loss tangent $(\tan \delta)$ versus frequency at various temperatures as shown in Fig. 3 for PEIL08. The rising trend of $\tan \delta$ at low frequencies is representative of the presence of DC conductivity in the polymer electrolyte system. At low frequencies, $\tan \delta$ increased due to the active compound (ohmic) being more dominant than the reactive component (capacitive). At higher frequencies, $\tan \delta$ decreased with frequency since the ohmic portion is independent of frequency and the reactive compound increased in proportion to the frequency [16].

The shift of $\tan \delta$ peaks to higher frequencies for samples at higher temperatures indicate that the charge carriers/ions are thermally activated and the highest conducting sample (343K) has the lowest peak intensity. The occurrence of relaxation time, $\tau$ can be obtained from the peak of $\tan \delta$ using the relation [17]:

$\tau=1 / \omega_{\text {peak }}$

where, $\omega=2 \pi f$ and $f$ is the frequency of the relaxation peak.

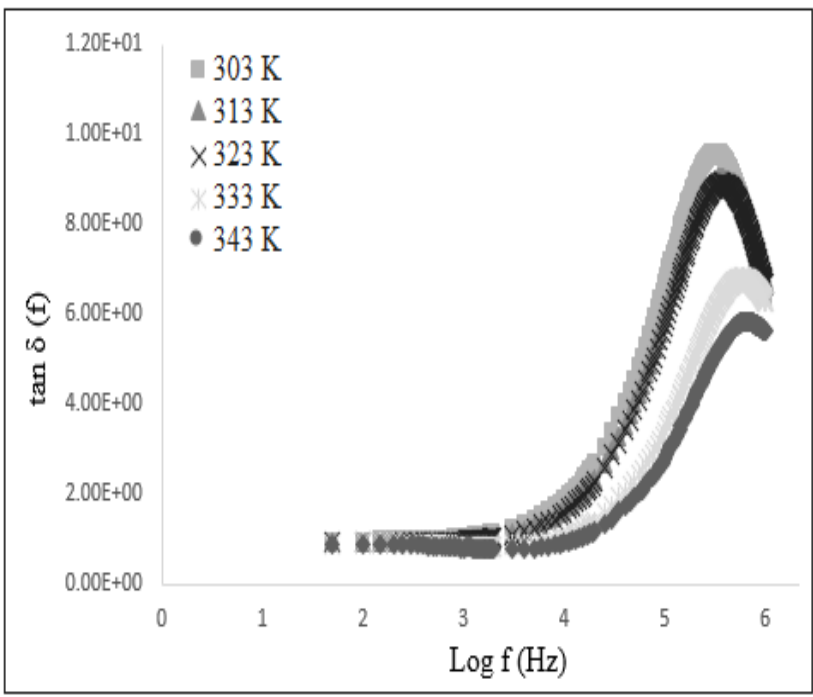

Fig. 3: Variation of loss tangent with frequency at various temperatures.

Table 1 shows the relaxation time at each temperature and from this table, it is observed that the highest temperature (343 K) achieved the lowest relaxation time due to increase in number of ions and increase in mobility of ions in the polymer system, which in turn decreased the relaxation time. The results show that relaxation is a thermally activated process where the relaxation time decreased when temperature increased. 
Table 1: Conductivity, $\sigma$ and Relaxation Time, $\tau$ for PEO-LiTFSI-EMImTFSI at 8 wt. $\%$ of EMIm-TFSI as a Function of Temperature.

\begin{tabular}{ccc}
\hline Temperature $(\mathbf{K})$ & $\begin{array}{c}\text { Relaxation Time, } \boldsymbol{\tau} \\
(\mathbf{s})\end{array}$ & $\begin{array}{c}\text { Conductivity, } \boldsymbol{\sigma}\left(\mathbf{S} / \mathbf{c m}^{-}\right. \\
\mathbf{1})\end{array}$ \\
\hline 303 & $3.84 \times 10^{-7}$ & $1.44 \times 10^{-3}$ \\
313 & $3.66 \times 10^{-7}$ & $2.05 \times 10^{-3}$ \\
323 & $3.50 \times 10^{-7}$ & $2.80 \times 10^{-3}$ \\
333 & $2.67 \times 10^{-7}$ & $5.13 \times 10^{-3}$ \\
343 & $1.99 \times 10^{-7}$ & $7.10 \times 10^{-3}$ \\
\hline
\end{tabular}

These results may be corroborated by the graph of $\varepsilon_{\mathrm{r}}$ and $\varepsilon_{\mathrm{i}}$ versus temperature and $\mathrm{M}_{\mathrm{r}}$ and $\mathrm{M}_{\mathrm{i}}$ versus temperature as shown in Fig. 4 and Fig. 5.

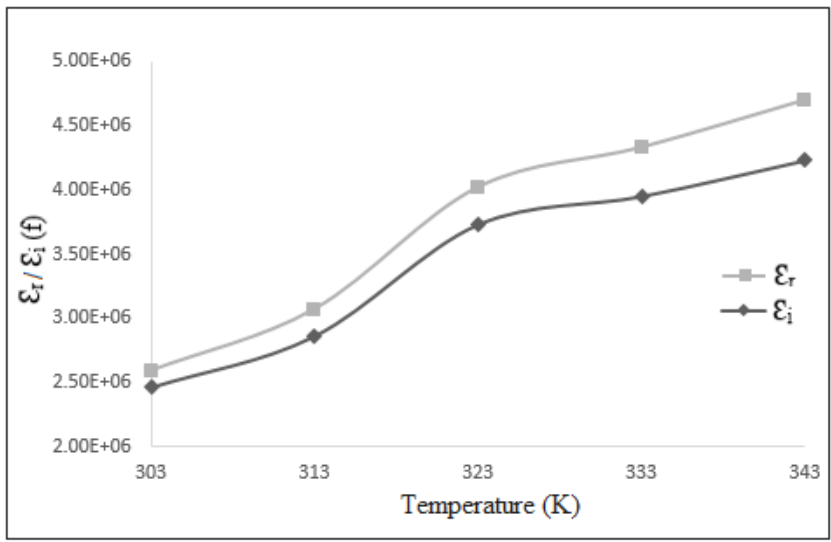

Fig. 4: $\varepsilon_{\mathrm{r}}$ and $\varepsilon_{\mathrm{i}}$ versus temperature

From the graph of temperature dependence, at low temperature, the charge carriers cannot orient themselves with respect to the direction of applied field [18], as they did not get enough thermal energy. When the temperature increased, it led to dissociation of more ions that caused increase in the number of free ions [19]. The charge carriers also get enough thermal energy to be excited and hence, they were able to obey the change in the external field and contributed to polarization, which in turn led to an increase of dielectric constant and dielectric loss value in the samples [20]. This is due to more energy being dissipated. The highest ionic conductivity value at $343 \mathrm{~K}$, thus has the highest $\varepsilon_{\mathrm{r}}$ and $\varepsilon_{\mathrm{i}}$ proving that $\varepsilon_{\mathrm{r}}$ and $\varepsilon_{\mathrm{i}}$ are thermally activated [21] and the lowest $\mathrm{M}_{\mathrm{r}}$ and $\mathrm{M}_{\mathrm{r}}$ showing the spread of conductivity relaxation, an indication of temperature-dependence relaxation phenomenon [15]. The shift of $\mathrm{M}_{\mathrm{r}}$ and $\mathrm{M}_{\mathrm{i}}$ towards the higher frequency side also implies that the thermally activated ionic conduction relaxation is due to hopping of charge carriers [15].

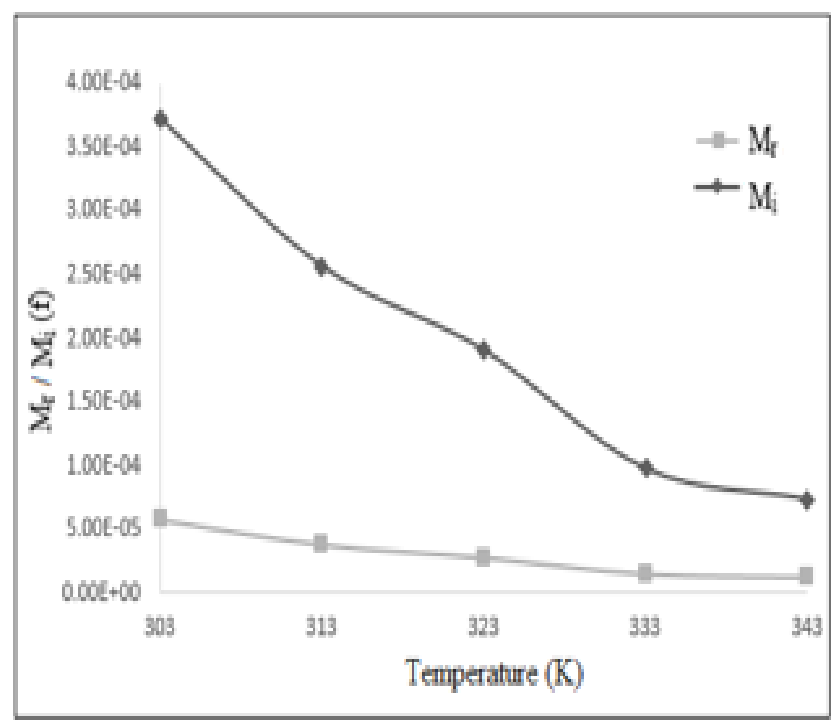

Fig. 5: $\mathrm{M}_{\mathrm{r}}$ and $\mathrm{M}_{\mathrm{i}}$ versus temperature

\section{Conclusion}

The electrical relaxation process occurring in PEO-LiTFSIEMIMTFSI system has been found to be temperature and frequency dependent. The lowest relaxation time is achieved at the highest temperature, which is $343 \mathrm{~K}$.

\section{Acknowledgement}

The authors would like to thank the Ministry of Higher Education for the Sports Scholarship awarded and Faculty of Applied Sciences, Universiti Teknologi MARA for the opportunity given.

\section{References}

[1] Li W, Pang Y, Liu J, Liu G, Wang Y \& Xa Y, A PEO-based Gel Polymer Electrolyte for Lithium Ion Batteries, RSC Advances, Vol. 7, (2017), pp:23494-23501, DOI: https://doi.org/10.1039/C7RA $02603 \mathrm{~J}$

[2] Ngai KS, Rames S, Ramesh K, \& Jong CJ, A Review of Polymer Electrolytes: Fundamental, Approaches and Applications, Ionic, Vol.8, Issue 8, (2016), pp:1259-1279, DOI: https://doi.org/10.1007/ s1158-016-1756-4

[3] Ing. Vitezslav H, Polymer Gel Electrolytes, Department of Electrical and Electronic Technology, (2009)

[4] Abdullah, SZ, Structural Electrical and Thermal Studies of PEOBased Polymer Electrolytes, Universiti Teknologi Mara, (2010)

[5] Yun-Sheng Y, Rick J, \& Bing-Joe H, Ionic Liquid Polymer Electrolytes, Journal of Material Chemistry. A, Vol. 1, (2013), pp:27192743.

[6] Shojaatalhosseini M, Elamin K, \& Swenson J, Conductivity and Relaxation in Polymer Based Solid Electrolyte, The Journal of Physical Chemistry B, Vol. 121, Issue.41, (2017), pp:9699-9707.

[7] Bircan H, Seshadri V, Padilla J, Invernale M, Otero TF, \& Asotzing G, Use of Polymer/Ionic Liquid Plasticizer as Gel Polymer Electrolytes in Electrochromic Devices Journal of Physics: Conference Edition, (2008)

[8] Aziz SB, Woo TJ, Kadir MFZ \& Ahmed HM, A Conceptual Polymer Electrolytes and Ion Transport Models, Journal of Science: Advanced Materials \& Devices, Vol. 3, Issue 1, (2018), pp:1-17, DOI: https://doi.org/10.1016/j.jsamad.2018.01.002

[9] Azmar A, Razamin NAY \& Tan W, Properties of Temperature on Conductivity and Dielectric Properties of PMA / PVAc Blend with Addition of TPAI Salt, Material Science Forum, Vol. 889, (2017), pp:201-206, DOI: https://doi.org/10.4028/www .scientific.net/ MSF .889.201

[10] Ramesh S \& Liew CW, Dielectric and FTIR Studies on Blending of [xPMMA-(1-x) PVC] with LiTFSI, Measurement, Vol. 46, Issue. 5, (2013), pp: https://doi.org/10.1016/j.measurement.2013.01.003

[11] Dineshkumar S, Muthusamy A \& Chandrasekaran J, Temperature \& Frequency Dependent Dielectric Properties of Electrically Conducting Oxidatively Synthesized Polyazomethines and Their Structural, Optical and Thermal Characterizations, Journal of Molecular Structure, Vol. 1128, (2017), pp:730-740, DOI: https://doi.org/10.1016/j. molstruc.2016.09.051

[12] Ravi M, Pavani Y, Kiran Kumar K, Bhavani S, Sharma AK, \& Narashimo Rao VVR, Studies on Electrical and Dielectric Properties of PVP- $\mathrm{KBrO}_{4}$ Complexed Polymer Electrolyte Films, Materials Chemistry and Physics, Vol. 130, Issue. 1-2, (2011), pp:442-448, DOI: https://doi.org/ 10.1016/j.matchemphys.2011.07.06

[13] Sikkanthar S, Karthikeyan S, Selvasekarapandian S, Arunkumar D, \& Nithya H, Structural, Electrical Conducting and Transport Analysis of PAN-NH ${ }_{4}$ CI Polymer Electrolyte System, Ionics, Vol.22, Issue.7, (2016), pp:1085-1094, DOI: https://doi.org/10.1007/s11551016-1645-x

[14] Hashim H, Adam NI, Zaki NHM, Mahmud ZS, Said CMS, Yahya MZA, \& Ali AMM, Natural Rubber-Grafted with $30 \%$ Poly (methylmethacrylate) Characterization for Application on Lithium Polymer Battery, International Conference on Science and Social Research (CSSR 2010), DOI: https://10.1099/CSSR.2010.5773825

[15] Muhammad FH, Jamal A, \& Tan W, Dielectric and AC Conductivity Behaviour of Hexanoyl Chitosan-NaI Based Polymer Electrolytes, International Journal of Advanced and Applied Sciences, Vol. 3, Issue. 10, (2016), pp: 9-13 
[16] Woo HJ, Majid SR \& Arof AK, Dielectric Properties and Morphology of Polymer Electrolyte Based on Poly ( $\varepsilon$-caprolactone) and Ammonium Thiocyanate, Materials Chemistry and Physics, Vol. 134, Issue. 2-3, (2012), pp:755-761, DOI: https://doi.org/10.1016/j.matchemphys. 2012.03.064

[17] Gondaliya N, Konchan DK Sharma P, Jayswal MS, \& Pont M, Conductivity and Dielectric Behavior of $\mathrm{AgCF}_{3} \mathrm{SO}_{3}$ Doped PEO Polymer Films, Integrated Ferroelectrics, Vol. 119, Issue. 1, (2010), DOI: https://doi.org/10.1080/105545878.2010.489494

[18] Kaiser M, Magnetic ad Dielectric Properties of Low Vanadium Doped Nickel-Zinc-Copper Ferrites, Journal of Physics and Chemistry of Solids, Vol 71, Issue. 10, (2010), pp:1451-1457, DOI: https://doi.org/ 10.1016/j.jpcs.2010.07.011

[19] Muhammad FH, Subban RHY, Majid SR, Tan W, \& Arof AK, Characterisation of $\mathrm{Al}_{2} \mathrm{O}_{3}$ Doped Hexanoyl Chitosan- $\mathrm{LiCF}_{2} \mathrm{SO}_{3}-\mathrm{EC}$ Polymer Electrolytes, Material Research Innovations, Vol. 13, Issue. 3, (2009), pp:249-251, DOI: doi.org/10.1179/143307509x440433

[20] Farea AMM, Kumar S, Batoo KM, Yousef A, \& Alimuddin, Influence of Frequency, Temperature and Composition on Electrical Properties of Polycrystalline $\mathrm{CO}_{0.5}\left(\mathrm{~d}_{\mathrm{x}} \mathrm{FE}_{2.5-\mathrm{x}} \mathrm{O}_{4}\right)$ Ferrites, Physica B Condensed Matter, Vol. 403, Issue. 4, (2008), pp:684-701, DOI: https://doi.org/ 10.1016/j.physb.2007.09.080

[21] Gurusiddappa J, Madhuri W, Suvarna RP, \& Dasan KP, Studies on the Morphology and Conductivity of $\mathrm{PEO} / \mathrm{LiCIO}_{4}$, Materials: Proceedings, Vol. 3, Issue. 6, (2016), pp:1451-1459, DOI https://doi.org/10.1016/2016.04.028 